\title{
ICBrainDB: database with EEG activity and associated gene mutations
}

\author{
Ivanov R.A. ${ }^{2 *}$, Kazantsev F.V. ${ }^{1,2,3}$, Klimenko A.I. ${ }^{1,2,3}$, Zavarzin E.A. ${ }^{1}$, \\ Saprigyn A.E. ${ }^{4}$, Matushkin Y.G. ${ }^{1,2}$, Savostyanov A.N. ${ }^{1,2,5}$, Lashin S.A. ${ }^{1,2,3}$ \\ ${ }^{1}$ Novosibirsk State University, Novosibirsk, Russia \\ ${ }^{2}$ Institute of Cytology and Genetics, SB RAS, Novosibirsk, Russia \\ ${ }^{3}$ Kurchatov Genomic Center of the Institute of Cytology and Genetics, SB RAS, Novosibirsk, Russia \\ ${ }^{4}$ Laboratory of Psychological Genetics of the Institute of Cytology and Genetics, SB RAS, \\ Novosibirsk, Russia \\ ${ }^{5}$ Institute of Neuroscience and Medicine, Novosibirsk, Russia \\ *ivanovromanart@bionet.nsc.ru
}

Key words: depression, database, EEG, SNP, questionnaires

Motivation and Aim: In the past decade, there has been a significant increase in the number of studies looking for associations between genetic factors and brain activity data from fMRI and EEG technologies. A considerable number of genes and polymorphisms associated with features of brain activity, which are related to depression susceptibility, have been found. However, so far there has been no systematization of this information in a convenient form for the subsequent analysis. We have developed an Internet resource - ICBrainDB that includes a database with information about patients, their mutations in genes, the results of test questionnaires, and EEG data of these patients.

Methods and Algorithms: The database is implemented with PostgreSQL. Data access is implemented in Python by REST API technology using FLASK and SQLAlchemy libraries. The API is available by the next address: http://icbraindb.cytogen.ru/api-v2/ Results: The database contains data about 1000 subjects, over 6300 mutations in 133 genes that associated with brain activity. The results of 17 test questionnaries in addition to more than 40000 files related to EEG analysis are available too. These data one can access by the software tools that are widely used in Artificial Neural Networks analysis (Python, R, Matlab).

Conclusion: Thus, ICBrainDB is a unique source of biomedical data that is extensively used for the search of associations between genotype and phenotype on both neurophysiological and behavioral levels. Moreover, it can be utilized for machine learning while solving various problems such as classification, regression and decision making based on the data available at the resource.

Acknowledgements: The research was partially funded by the RFBR Grant 18-29-13027 and the Budget Project 0259-2019-0008. 